\title{
Highly efficient quasi-static water desalination using monolayer graphene oxide/titania hybrid laminates
}

\author{
Pengzhan Sun ${ }^{1}$, Qiao Chen ${ }^{1,2}$, Xinda $\mathrm{Li}^{3}$, He Liu ${ }^{3}$, Kunlin Wang ${ }^{1}$, Minlin Zhong ${ }^{1}$, Jinquan Wei ${ }^{1}$, Dehai Wu ${ }^{3}$, \\ Renzhi $\mathrm{Ma}^{4}$, Takayoshi Sasaki ${ }^{4}$ and Hongwei $\mathrm{Zhu}^{1,2}$ \\ By intercalating monolayer titania (TO) nanosheets into graphene oxide (GO) laminates with mild ultraviolet (UV) reduction, the \\ as-prepared RGO/TO hybrid membranes exhibit excellent water desalination performances. Without external hydrostatic \\ pressures, the ion permeations through the RGO/TO hybrid membranes can be reduced to $<5 \%$ compared with the GO/TO \\ cases, while the water transmembrane permeations, which are measured using an isotope-labeling technique, can be retained up \\ to $\sim 60 \%$. The mechanism for the excellent water desalination performances of the RGO/TO hybrid laminates is discussed, which \\ indicates that the photoreduction of GO by TO is responsible for the effective rejection of ions, while the photoinduced \\ hydrophilic conversion of TO under UV irradiation is responsible for the well-retained water permeabilities. These excellent \\ properties make RGO/TO hybrid membranes favorable for practical water desalination.
}

NPG Asia Materials (2015) 7, e162; doi:10.1038/am.2015.7; published online 27 February 2015

\section{INTRODUCTION}

Currently, water desalination is of crucial importance because of the serious lack of clean and safe fresh water resources. ${ }^{1,2}$ The recently developed graphene-based nanomaterials ${ }^{3}$ may have the opportunity to make a special contribution to the development of membranebased water desalination technology. Typically, defect-free monolayer graphene film is impermeable to most gases and liquids in spite of its single-atom layer thickness. ${ }^{4}$ However, after introducing nanoscale pores with various sizes, shapes and functionalities into the matrix, the resulting nanoporous graphene membranes exhibit excellent selectivity toward various gases and ions, which is favorable for gas separation and water desalination. ${ }^{5-7}$ Unfortunately, wafer-scale single-crystal graphene membranes have not been produced thus far, and the irregular nanopores introduced by the current immature techniques cause significant stress concentrations in the matrix, which significantly degrades the mechanical strength of the nanoporous graphene membranes. These disadvantages limit the practical applications of nanoporous graphene films in water desalination, and the investigations on this aspect currently rely mostly on simulations. ${ }^{6,7}$

Conversely, graphene oxide (GO), which is synthesized using the modified Hummers' method, ${ }^{8}$ is another promising graphene derivative and can potentially be used in practical industrial applications due to its characteristics of low cost and ease of production on a large scale. $^{9-13}$ Scientists are now exploring the potential applications of GO in areas such as waste water treatment and water desalination. In view of the structure of the single-layer $\mathrm{GO}$ sheet, it can be treated as numerous $s p^{2}$ graphitic nanoregions clustered on the $s p^{3} \mathrm{C}-\mathrm{O}$ matrix. ${ }^{14,15}$ When large amounts of GO sheets are stacked together, the strong interlayer hydrogen bonds hold them together to form a freestanding laminate with sufficient mechanical strength for manipulation. ${ }^{16}$ Within the laminate, the $s p^{2}$ graphitic clusters are connected together across all the stacking layers to form a network of nanocapillaries through which water can experience an ultrafast and unimpeded transmembrane permeation while other liquids and gases are completely blocked. ${ }^{16,17}$ Notably, due to the narrow dimension of the nanocapillaries and the co-existence of $s p^{2}$ aromatic channels with various oxygen functionalities, the $\mathrm{GO}$ membranes can afford excellent selectivity toward various ions based on the molecular sieving effect ${ }^{18}$ and diverse chemical interactions, ${ }^{19-22}$ which is favorable for filtration and separation. However, in regard to water desalination, assynthesized GO membranes cannot perform well at the current state due to rapid ion flows. ${ }^{18-20}$ For example, recent studies have revealed that GO-based membranes can only afford a salt rejection of $<40 \%$ towards $\mathrm{NaCl}, 23,24$ which is fairly poor and far from practical. More recently, $\mathrm{Mi}^{25}$ has proposed that for applications of $\mathrm{GO}$ membranes in water desalination, the GO interlayer spacing within the laminates has to be reduced to less than $0.7 \mathrm{~nm}$ to sieve the hydrated $\mathrm{Na}^{+}$ions (hydrated radii: $0.36 \mathrm{~nm}$ ) from water. However, previous results have also demonstrated that if the interlayer spacing is reduced to $\leqslant 0.6 \mathrm{~nm}$, the nanocapillaries cannot be filled, and water cannot permeate through completely. ${ }^{16}$ Such a small tunable range remains a challenge for the existing thermal and chemical reduction routes, and Nair et al. ${ }^{16}$ demonstrated that with the reduction of the GO membranes, the permeation of water is significantly weakened. If the GO

${ }^{1}$ School of Materials Science and Engineering, State Key Laboratory of New Ceramics and Fine Processing, Key Laboratory of Materials Processing Technology of MOE, Tsinghua University, Beijing, China; ${ }^{2}$ Center for Nano and Micro Mechanics, Tsinghua University, Beijing, China; ${ }^{3}$ Department of Mechanical Engineering, Tsinghua University, Beijing, China and ${ }^{4}$ International Center for Materials Nanoarchitectonics, National Institute for Materials Science, Ibaraki, Japan

Correspondence: Professor H Zhu, School of Materials Science and Engineering, State Key Laboratory of New Ceramics and Fine Processing, Key Laboratory of Materials Processing Technology of MOE, Tsinghua University, Beijing 100084, China.

E-mail: hongweizhu@tsinghua.edu.cn

Received 12 July 2014; revised 4 December 2014; accepted 23 December 2014 
membranes are reduced thoroughly, neither the water nor the ions can permeate through, which results in impermeable barrier films; furthermore, their abilities as water desalination membranes are completely lost, as shown in the work of Su et al. ${ }^{26}$ Therefore, a new reduction method that can be precisely controlled should be applied, and it is crucial to design GO-based membranes capable of rejecting ions while preserving high water permeations.

Under this background, monolayer titania $\left(\mathrm{Ti}_{0.87} \mathrm{O}_{2}\right.$, simplified as TO) nanosheets, ${ }^{27,28}$ another new type of two-dimensional nanomaterial that is prepared by delaminating layered titanates into their molecular single sheets, ${ }^{29,30}$ are designed to intercalate into the GO sheets to form hybrid membranes for potential applications in water desalination based on the following reasons: (i) under ultraviolet (UV) irradiation, the GO can be mildly reduced to RGO by the photocatalytic properties of the TO, which is a controllable process without the use of high temperatures and harsh chemical agents; ${ }^{31,32}$ (ii) under UV irradiation, the efficient photoinduced hydrophilic conversion of the TO nanosheets within the laminates enables the adsorption of more water molecules into and through the membranes; ${ }^{32-34}$ and (iii) the intercalation of the TO nanosheets into the GO layers may have an important role in adjusting the interlayer spacing. These characteristics of the TO nanosheets may thus help settle the contradiction between ion rejection and water permeation. Therefore, in this study, the GO/TO hybrid laminates were prepared using a facile vacuum-filtration method. After UV reduction, the water desalination properties of the RGO/TO hybrid membranes were investigated using a novel isotope-labeling technique.

\section{EXPERIMENTAL PROCEDURE}

\section{Preparation of GO(RGO)/TO hybrid membranes}

The GO flakes were synthesized using the modified Hummers' method starting from natural graphite and were then exposed to concentrated sulfuric acids, sodium nitrite and potassium permanganate. ${ }^{8}$ The TO nanosheets were synthesized by chemically delaminating layered titanates based on our previous study. ${ }^{29,30}$ After the synthesis procedures, the as-prepared GO and TO sheets were redispersed in water using sonication to form colloidal aqueous solutions $\left(0.1 \mathrm{mg} \mathrm{ml}^{-1}\right.$ for $\mathrm{GO}$ and $0.08 \mathrm{mg} \mathrm{ml}^{-1}$ for TO), mixed in equivalent volumes $(25 \mathrm{ml}$ for each) and vacuum-filtered with cellulose microfilters $(80 \%$ in porosity, $220 \mathrm{~nm}$ in pore diameter). The concentration of the TO solution was further varied to investigate the effects of the TO contents within the hybrid laminates on the water desalination performances. Then, the as-prepared GO/ $\mathrm{TO}$ membranes were dried in air at $55^{\circ} \mathrm{C}$ for $24 \mathrm{~h}$ before being used to enhance the adhesion between the membranes and the microfilters. Finally, they were exposed to UV irradiation (ZF7-C, $6 \mathrm{~W}, 254$ and $365 \mathrm{~nm}$ ) for various periods.

\section{Permeation experiments}

The ion permeation experiments were performed using a homemade apparatus, as indicated in Supplementary Figure S1a. Briefly, a plastic plate with a leak hole ( $5 \mathrm{~mm}$ in diameter) in the center separated the source reservoir from the drain reservoir. A piece of the GO/TO hybrid membrane with the cellulose microfilter underneath was sealed with double-faced copper tape onto the leak hole. The copper tape was also introduced with the same-sized aperture $(5 \mathrm{~mm}$ in diameter) so that the membrane could directly contact the source and drain solutions and facilitate ion transmembrane permeations (Supplementary Figure S1a). In the permeation experiments, $90 \mathrm{ml}$ of a $0.1-\mathrm{mol}^{-1}$ salt solution and deionized water was injected into the source and drain vessels, respectively, at the same speed. To avoid possible concentration gradients, mild magnetic stirrings were applied to both the source and the drain solutions, as indicated in Supplementary Figure S1b. During the ion permeation process, equivalent amounts $(0.5 \mathrm{ml})$ of the source and drain solutions were removed at regular intervals $(30 \mathrm{~min}$ ) to avoid external hydrostatic pressures across the membrane, and the drain solutions were exposed to an atomic emission spectroscopy for accurate cation concentrations.

\section{$\mathrm{D}_{2} \mathrm{O}$-labeled ion permeation experiments}

To investigate the water transport behaviors through the GO(RGO)/TO hybrid membranes during ion permeations, the source solutions were labeled with deuterium oxide $\left(\mathrm{D}_{2} \mathrm{O}\right)$ tracers. The permeation behavior of $\mathrm{D}_{2} \mathrm{O}$ was used to extrapolate that of water. In the experiments, $100 \mathrm{ml}$ of $0.1 \mathrm{moll}^{-1} \mathrm{NaCl}$ at $50 \mathrm{wt} \%$ of the $\mathrm{D}_{2} \mathrm{O}$ solution and deionized water was injected into the source and drain reservoirs, respectively. During the permeation processes, $0.5 \mathrm{ml}$ of the source and drain solutions was removed every $2 \mathrm{~h}$, and the drain solutions were exposed to an Fourier transform infrared (FTIR) and atomic emission spectroscopy for accurate concentrations of the $\mathrm{D}_{2} \mathrm{O}$ and $\mathrm{Na}^{+}$ions.

\section{Characterizations}

As-synthesized GO and TO flakes were characterized using atomic force microscopy (Agilent 5100, Santa Clara, CA, USA). The as-prepared GO/TO hybrid membranes were characterized using an optical microscope (ZEISS, Axio Scope.A1, Oberkochen, Germany), X-ray photoelectron spectroscopy (Ulvac-Phi, PHI Quantera SXM, AlKo, Kanagawa, Japan) and X-ray diffraction (Siemens, 08DISCOVER, $\lambda=0.15405 \mathrm{~nm}$, Munich, Germany). During the ion permeation experiments, the concentrations of the cations in the drain solutions were measured using atomic emission spectroscopy (Thermo Fisher Scientific, IRIS Intrepid II, Waltham, MA, USA), and the concentrations of the $\mathrm{D}_{2} \mathrm{O}$ tracers in the drains were measured using FTIR spectroscopy (Thermo Fisher Scientific, Nicolet 6700FTIR).

\section{RESULTS AND DISCUSSION}

GO flakes were synthesized using the modified Hummers' method starting from natural graphite, ${ }^{8}$ whereas the monolayer TO nanosheets were prepared by a chemical exfoliation of layered titanates based on our previous method. ${ }^{29,30}$ Figures $1 \mathrm{a}$ and $\mathrm{b}$ provide the atomic force microscopy characterizations of the as-synthesized GO and TO flakes, respectively, which reveal that the GO and TO sheets are single layered. After using sonication to dissolve these two types of flakes in water to form colloidal aqueous solutions (for example, $0.1 \mathrm{mg} \mathrm{ml}^{-1}$ for GO, $0.08 \mathrm{mg} \mathrm{ml}^{-1}$ for TO, Figures $1 \mathrm{c}$ and $\mathrm{d}$ ), they were mixed in equivalent volumes $(25 \mathrm{ml}$, Figure 1e) followed by vacuum-filtration to form uniform GO/TO hybrid membranes (Figure 1f). In an aqueous solution, the ionization of the oxygen-containing functional groups charges the GO flakes negatively; due to the presence of $\mathrm{Ti}$ vacancies $\left(\mathrm{Ti}_{0.87} \mathrm{O}_{2}\right)$, the as-synthesized $\mathrm{TO}$ nanosheets are also negatively charged. Therefore, mixing these two types of flakes together in water should give rise to a stable aqueous suspension, without obvious aggregation, and further result in the formation of uniform GO/TO hybrid laminates by vacuum-filtration, as indicated in Figures 1e and f. For the as-prepared GO/TO membranes, electronhole pairs will be generated in TO nanosheets under UV irradiation. Then, the electrons will be captured by the GO and lead to a further reduction in the GO sheets, as illustrated in Figure 1g. This photocatalytic reduction procedure is mild and controllable and can be used to tune the internal structure of the GO/TO membranes. Figure $1 \mathrm{~h}$ presents the photographs of the GO/TO membranes after UV irradiation for various degrees. The figure reveals that the color of the hybrid membrane gradually changes from brown to black with UV irradiation, which indicates the gradual reduction of the GO within the hybrid laminates. Using these GO(RGO)/TO membranes, the ion transmembrane transport properties were investigated, as sketched in Figure 1i.

The ion permeation experiments were conducted using a homemade permeation apparatus, as shown in Supplementary Figure S1a. A piece of the $\mathrm{GO}(\mathrm{RGO}) / \mathrm{TO}$ membrane with the cellulose microfilter underneath was sealed with double-sided copper tape onto the leak hole $(5 \mathrm{~mm}$ in diameter) that separated the source reservoir from the drain reservoir. It should be noted that the cellulose microfilters 

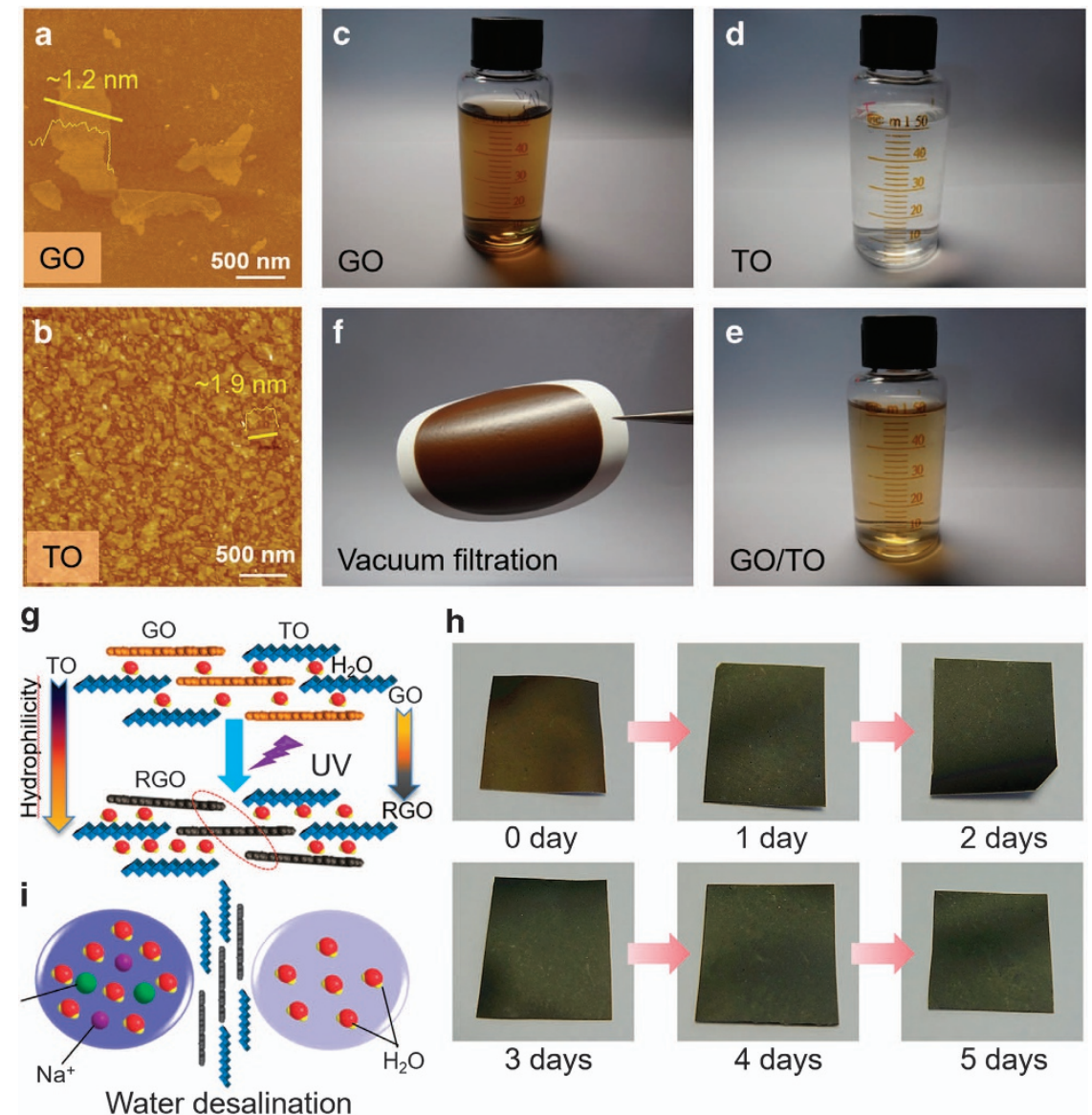

Figure 1 AFM characterizations of as-synthesized (a) graphene oxide (GO) and (b) titania (TO) nanosheets. Photographs of (c) $0.1 \mathrm{mg}^{-1} \mathrm{GO}^{-1}$ (d) $0.08 \mathrm{mg} \mathrm{ml}^{-1} \mathrm{TO}$ and (e) GO/TO hybrid solutions mixed in equivalent volumes and (f) GO/TO hybrid membranes prepared by vacuum-filtration. (g) Schematic diagram for the reduction of GO by TO within the hybrid membranes under ultraviolet (UV) irradiation. (h) Photographs of the GO/TO membranes after UV irradiation. (i) Schematic diagram for the water desalination process using RGO/TO hybrid membranes.

underneath were not detached from the hybrid membranes because the polymeric substrates could provide a valuable mechanical strength enhancement for the GO/TO membranes. The continuity and intactness of the membranes were checked before and after the permeation experiments using an optical microscope to ensure the validity of the runs. Initially, $90 \mathrm{ml}$ of a $0.1-\mathrm{mol}^{-1} \mathrm{NaCl}$ solution and deionized water was injected into the source and drain vessels, respectively. Mild magnetic stirrings were applied to both the source and the drain solutions to avoid possible concentration gradients, as indicated in Supplementary Figure S1b. During the ion permeation process, equivalent amounts $(0.5 \mathrm{ml})$ of the source and drain solutions were removed at regular intervals $(30 \mathrm{~min})$ to avoid external hydrostatic pressures across the membrane, and the drain solutions were exposed to an atomic emission spectroscopy for accurate cation concentrations, which were subsequently plotted as a function of time to reflect the permeation behaviors of the source ions through the $\mathrm{GO} / \mathrm{TO}$ hybrid laminates with various extents of UV reduction, as indicated in Figure 2.

First, $25 \mathrm{ml}$ of $0.1 \mathrm{mg} \mathrm{ml}^{-1} \mathrm{GO}$ and $0.08 \mathrm{mg} \mathrm{ml}^{-1} \mathrm{TO}$ solutions were mixed together and vacuum-filtrated to form the GO/TO hybrid membranes (named as 'GO0.1/TO0.08'). After undergoing UV irradiation for $0-4$ days, the $\mathrm{Na}^{+}$ion permeations through these membranes are plotted in Figure 2a, and the ion permeation rates can be extracted, as indicated in Figure $2 \mathrm{~b}$. The results indicate that with UV reduction, the permeabilities of the $\mathrm{Na}^{+}$ions are reduced sharply.
For the membrane after 3-day UV irradiation, the ion rejection can be up to $>95 \%$ compared with the unirradiated case, which is favorable for water desalination. However, with further doses of irradiation, the ion permeability is gradually enhanced, which can be attributed to the introduction of extra defects in the GO flakes under longer periods of UV irradiation that facilitate ion permeations. ${ }^{35}$ Similar phenomena have also been found from the transmembrane permeations of the $\mathrm{MgCl}_{2}$ and $\mathrm{Na}_{2} \mathrm{SO}_{4}$ sources, as presented in Supplementary Figure S2. Next, the ratio of the TO nanosheets within the membranes was further varied to investigate its effect on the ion rejection performance. In this respect, $25 \mathrm{ml}$ of $0.1 \mathrm{mg} \mathrm{ml}^{-1} \mathrm{GO}$ and $0.04 \mathrm{mg} \mathrm{ml}^{-1}$ TO (GO0.1/TO0.04) and $25 \mathrm{ml}$ of $0.1 \mathrm{mg} \mathrm{ml}^{-1} \mathrm{GO}$ and $0.16 \mathrm{mg} \mathrm{ml}^{-1}$ TO (GO0.1/TO0.16) were mixed and vacuum-filtrated to form GO/ TO membranes with various TO contents. The $\mathrm{Na}^{+}$ion permeations and the corresponding permeation rates through these two types of hybrid laminates after progressive UV reduction are provided in Figures $2 \mathrm{c}-\mathrm{f}$. The figures reveal that the ion permeations exhibit similar change tendencies compared with that of GO0.1/TO0.08; that is, with UV irradiation, the ion permeations decrease sharply at first and then increase slightly. However, the ion rejection performance of GO0.1/TO0.08 is clearly better than that of GO0.1/TO0.04 and GO0.1/TO0.16. For the GO0.1/TO0.04 membranes, the number of TO nanosheets within the membranes is insufficient for the full reduction of the GO flakes under the same dose of UV irradiation. However, for the GO0.1/TO0.16 membranes, the number of TO 

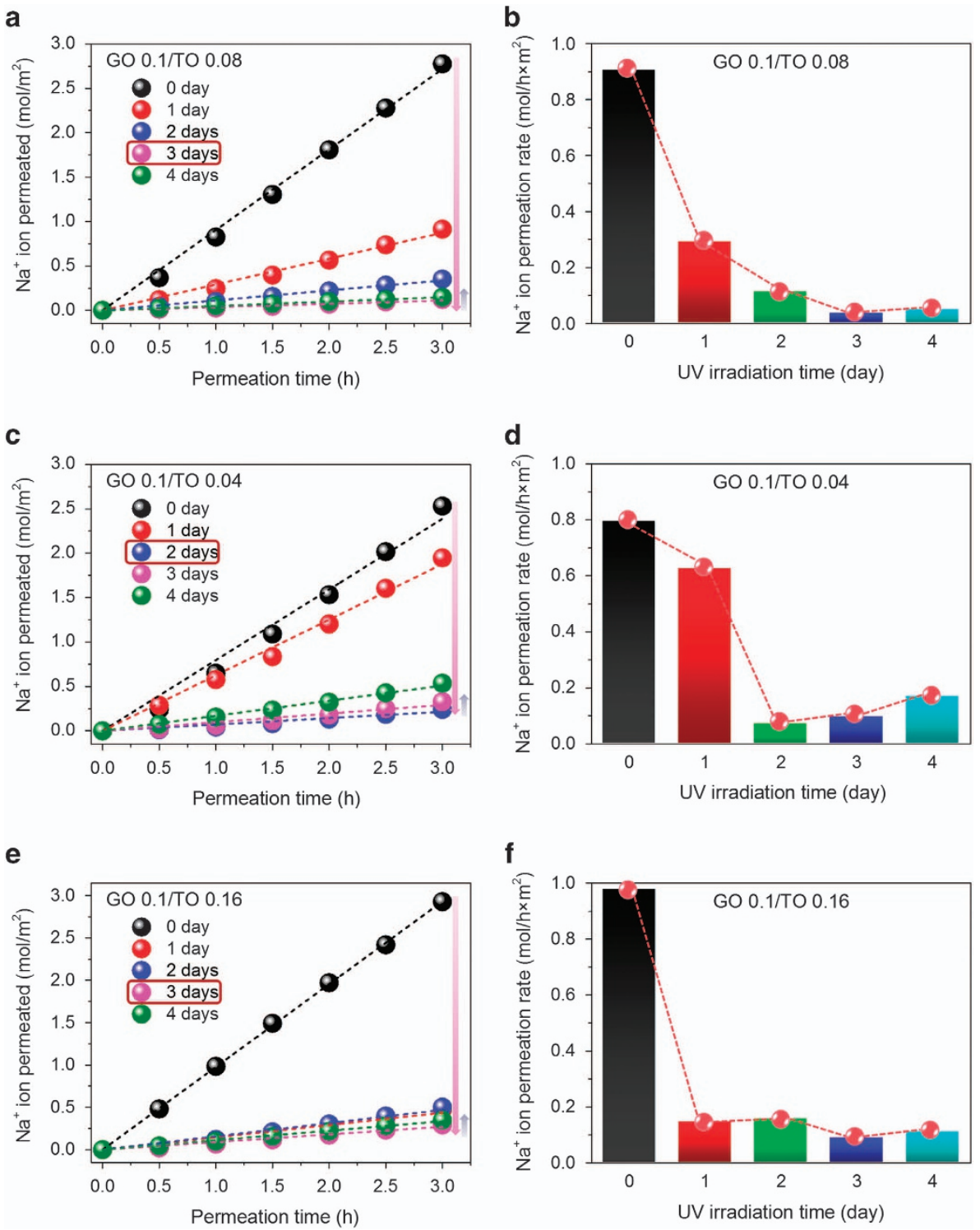

Figure 2 Transmembrane permeations of $0.1 \mathrm{moll}^{-1} \mathrm{NaCl}$ sources and corresponding permeation rates of (a, b) G00.1/T00.08, (c, d) G00.1/TO0.04 and (e, f) G00.1/TO0.16 membranes. GO, graphene oxide; TO, titania; UV, ultraviolet.

nanosheets is surplus, and the efficient photoinduced hydrophilic conversion of the TO under UV irradiation allows more ions and water molecules to transport into and through the hybrid membranes. These reasons may thus explain the poor ion rejection performances of GO0.1/TO0.04 and GO0.1/TO0.16 compared with the case of the GO0.1/TO0.08 membranes. Notably, for the pristine GO/TO hybrid laminates, the $\mathrm{Na}^{+}$ion permeations gradually increase with the TO contents in spite of the increase in the membrane thicknesses (Supplementary Figure S3). This result indicates that the more hydrophilic TO nanosheets within the membranes can adsorb more electrolytes into and through the membranes and further tune the mass transport properties of the GO/TO hybrids.

The topographies of GO0.1, GO0.1/TO0.04, GO0.1/TO0.08 and GO0.1/TO0.16 were characterized using dark-field optical microscopy, as indicated in Figure 3. Figures $3 \mathrm{a}-\mathrm{c}$ reveal that with a gradual increase in the TO content within the membranes, the number of wrinkles on the surfaces gradually decreases. Notably, for GO0.1/ TO0.08 (Figure 3c), few wrinkles can be observed on the surfaces.
However, with a further increase in the TO content, small wrinkles begin to reemerge. Combined with the ion permeation results in Figure 2, it can be concluded that the topographies of the hybrid laminates also affect the salt rejection performances: the flatter the membranes, the better salt rejection performances that can be obtained. Therefore, for the following experiments, unless otherwise specified, all water desalination experiments were conducted with GO0.1/TO0.08 hybrid laminates.

Next, the structure evolution of the GO/TO hybrid membranes under progressive UV irradiation was monitored using an X-ray photoelectron spectroscopy, as indicated in Figure 4. Figures $4 a-c$ reveal that with UV irradiation, the amount of oxygen functionalities decrease continuously, which leads to a gradual reduction of the GO flakes within the membranes. In contrast, for the Ti2 $p$ spectra provided in Figure $4 d$, the peaks located at $\sim 458.7$ and $\sim 464.5 \mathrm{eV}$, corresponding to the formation of the $\mathrm{Ti}^{4+}$ chemical state, do not shift with UV irradiation, which indicates the negligible structural distortion of the TO nanosheets during the photoreduction of the adjacent 

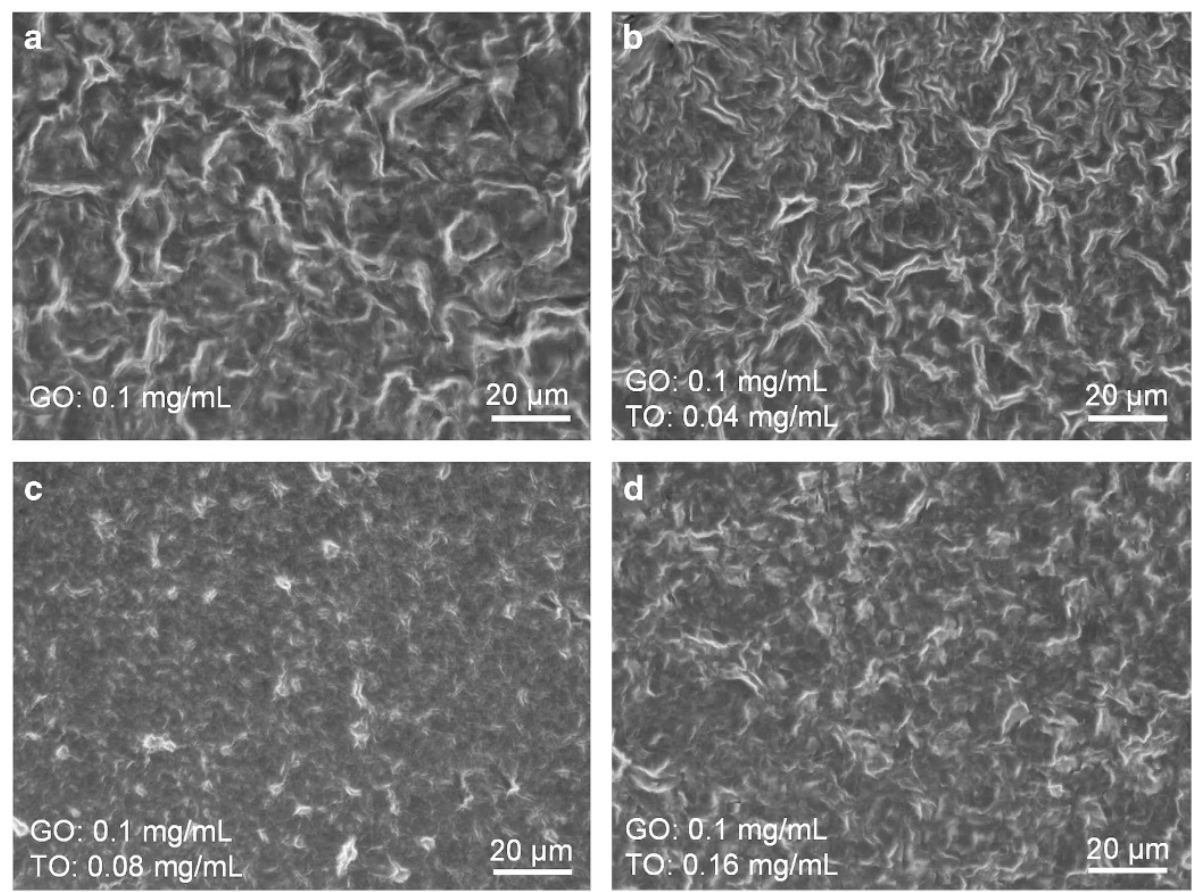

Figure 3 Dark-field optical images of (a) G00.1, (b) G00.1/T00.04, (c) G00.1/T00.08 and (d) G00.1/TO0.16. GO, graphene oxide; TO, titania.

GO flakes. ${ }^{36}$ Furthermore, the changes in the interlayer spacing of the $\mathrm{GO} / \mathrm{TO}$ hybrid membranes under UV irradiation were recorded using $\mathrm{X}$-ray diffraction, as indicated in Figure 5 . In the dry state, it is interesting to note that the interlayer spacing of the GO/TO laminates remains nearly unchanged with UV irradiation (Figure 5a). Generally, when the GO/TO samples are exposed to UV, the GO flakes within the membranes will be gradually reduced, which leads to a decrease in the interlayer spacing. However, due to the efficient photoinduced hydrophilic transformation of the TO nanosheets under UV irradiation, more water molecules will be intercalated in the membranes, and the average interlayer spacing will be retained, as illustrated in Figure 1g. In contrast, in regard to the fully wet state (Figure 5b), during the first 1-day UV irradiation, the interlayer spacing of the hybrid membrane remains nearly constant and is comparable to the dry states. However, with further irradiation of UV light, the interlayer spacing in the wet state has a sudden enhancement from $\sim 0.95$ to $\sim 1.22 \mathrm{~nm}$. These results are encouraging because under UV irradiation, the as-reduced RGO/TO membranes exhibit excellent ion rejection performances (Figures $2 \mathrm{a}$ and $\mathrm{b}$ ) while the TO sheets within the membranes become superhydrophilic ${ }^{33}$; furthermore, the interlayer spacing is enhanced, which allows more water molecules to transport into and through the hybrid membranes and may lead to the preservation of the relatively high water transmembrane permeation rates.

On the basis of the above results, the permeations of the source water through the GO/TO hybrids after UV irradiation for 0 and 3 days were studied using an isotope-labeling technique, as illustrated in Figure 6a. Measuring the water transmembrane permeations under zero external hydrostatic pressure is challenging because no macroscopic or microscopic differences between the sources and the drains can be detected. Therefore, $50 \mathrm{wt} \% \mathrm{D}_{2} \mathrm{O}$ was utilized as a tracer to label the $0.1-\mathrm{moll}^{-1} \mathrm{NaCl}$ source solution, and the permeation behavior of the $\mathrm{D}_{2} \mathrm{O}$ through the $\mathrm{GO}(\mathrm{RGO}) / \mathrm{TO}$ hybrid membrane was investigated to extrapolate that of water. To measure the concentration of the $\mathrm{D}_{2} \mathrm{O}$ in the drain solutions, FTIR spectroscopy was used. Figure $6 \mathrm{~b}$ presents a typical FTIR spectrum for a $50 \mathrm{wt} \%$ $\mathrm{D}_{2} \mathrm{O}$ solution. In the case of liquid $\mathrm{H}_{2} \mathrm{O}$, the extension vibration modes $\left(v_{1}\right.$ and $\left.v_{3}\right)$ overlap together to form a peak at $\sim 3440 \mathrm{~cm}^{-1}$, and the angular vibration mode $v_{2}$ is located at $\sim 1645 \mathrm{~cm}^{-1}$. In contrast, in the case of $\mathrm{D}_{2} \mathrm{O}$, due to the slightly larger mass of deuterium, the peak corresponding to the extension vibration modes $\left(v_{1}{ }^{\prime}\right.$ and $v_{3}{ }^{\prime}$ ) shifts to $\sim 2540 \mathrm{~cm}^{-1}$, and the peak corresponding to the angular vibration mode $v_{2}^{\prime}$ shifts to $\sim 1210 \mathrm{~cm}^{-1}$, as indicated in Figure $6 \mathrm{~b}$. Therefore, based on the intensity of the peak located at $\sim 2540 \mathrm{~cm}^{-1}$, which is first baselined and normalized with the peak located at $\sim 3440 \mathrm{~cm}^{-1}$, the $\mathrm{D}_{2} \mathrm{O}$ concentration in the drains can be calculated based on our recent study: $A=0.01983 c_{\mathrm{m}}$, where $A$ is the absorption intensity at $\sim 2540 \mathrm{~cm}^{-1}$ and $c_{\mathrm{m}}$ is the mass concentration of $\mathrm{D}_{2} \mathrm{O} .{ }^{37}$ For the permeation of $0.1 \mathrm{moll}^{-1} \mathrm{NaCl}$ solution containing $50 \mathrm{wt} \% \mathrm{D}_{2} \mathrm{O}$, it should be noted that in Figure $6 \mathrm{c}$, the water permeation through the RGO/TO hybrid membrane after 3-day UV irradiation is just slightly reduced compared with that of the pristine case. The water fluxes through these two types of membranes were extracted in Supplementary Figure S4a, and the water diffusion coefficients (normalized by the thickness of GO(RGO)/TO hybrid membranes, $\left.D_{\text {water }} / l\right)$ were calculated according to Fick's first law, which is applicable due to the tiny change in the drain concentration compared with the source $(<5 \%)$ : $D_{\text {water }} / l=J / \Delta c$, where $J$ is the water flux and $\Delta c$ is the concentration gradient across the membrane. As presented in Figure 6d, after UV reduction for 3 days, the water diffusion coefficients through the RGO/TO hybrid membranes can be retained up to $\sim 60 \%$ compared with the unreduced case, which demonstrates the excellent water permeability of the RGO/TO membranes. However, as indicated in Figure 6e, the ion permeation can be blocked almost entirely after the 3-day UV reduction, which demonstrates the excellent salt rejection ability of the RGO/TO membranes. The ion fluxes through the hybrid laminates after UV irradiation for 0 and 3 days were further calculated in Supplementary Figure S4b; based on these, the ion diffusion coefficients were obtained, as presented in Figure 6f. This figure reveals that the salt 

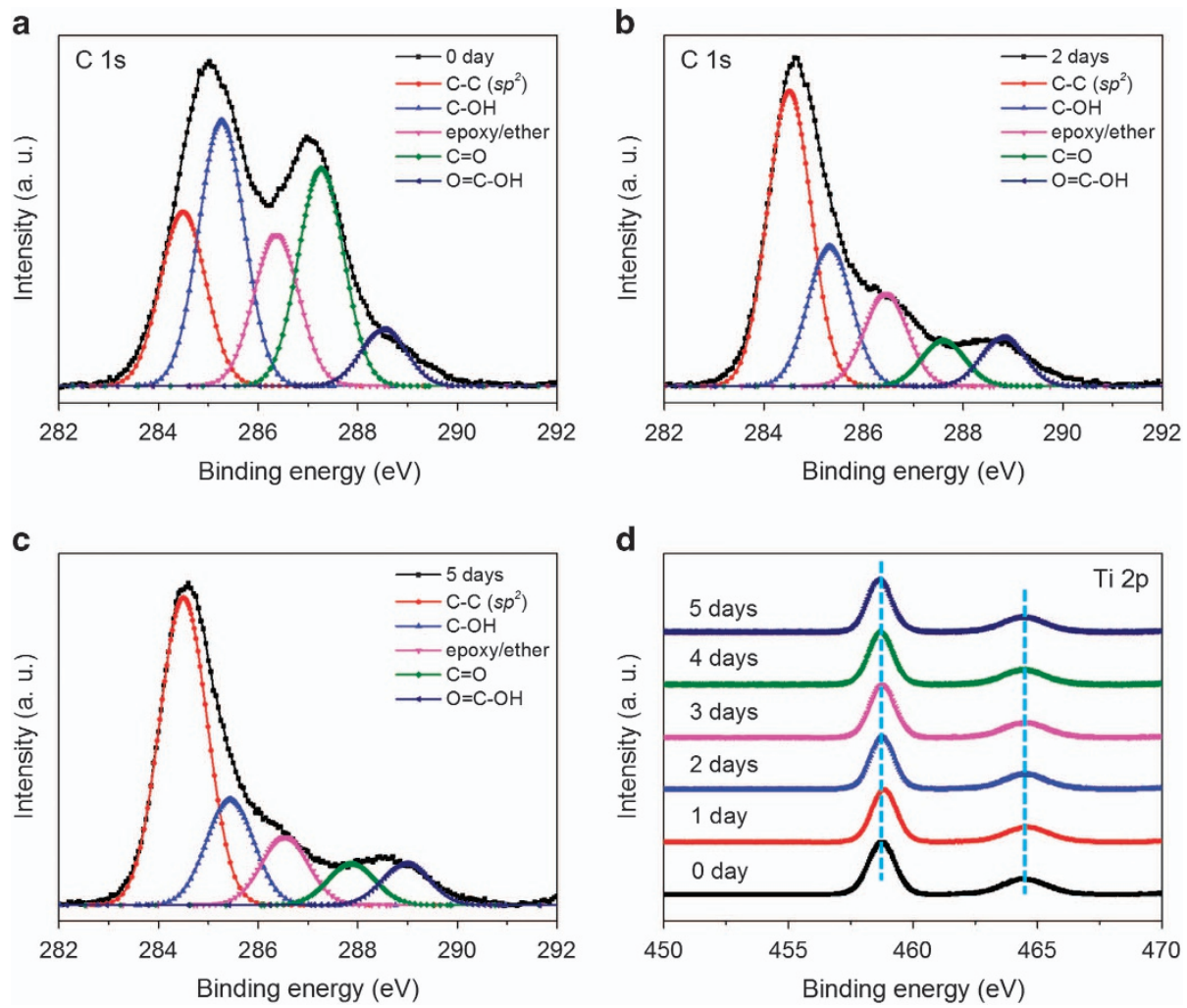

Figure $4 \mathrm{C}$ 1s X-ray photoelectron spectroscopy (XPS) spectra of G00.1/T00.08 membranes after ultraviolet (UV) irradiation for (a) 0 days, (b) 2 days and (c) 5 days. (d) Ti2p XPS spectra of G00.1/TO0.08 membranes after UV irradiation for 0-5 days. GO, graphene oxide; TO, titania.
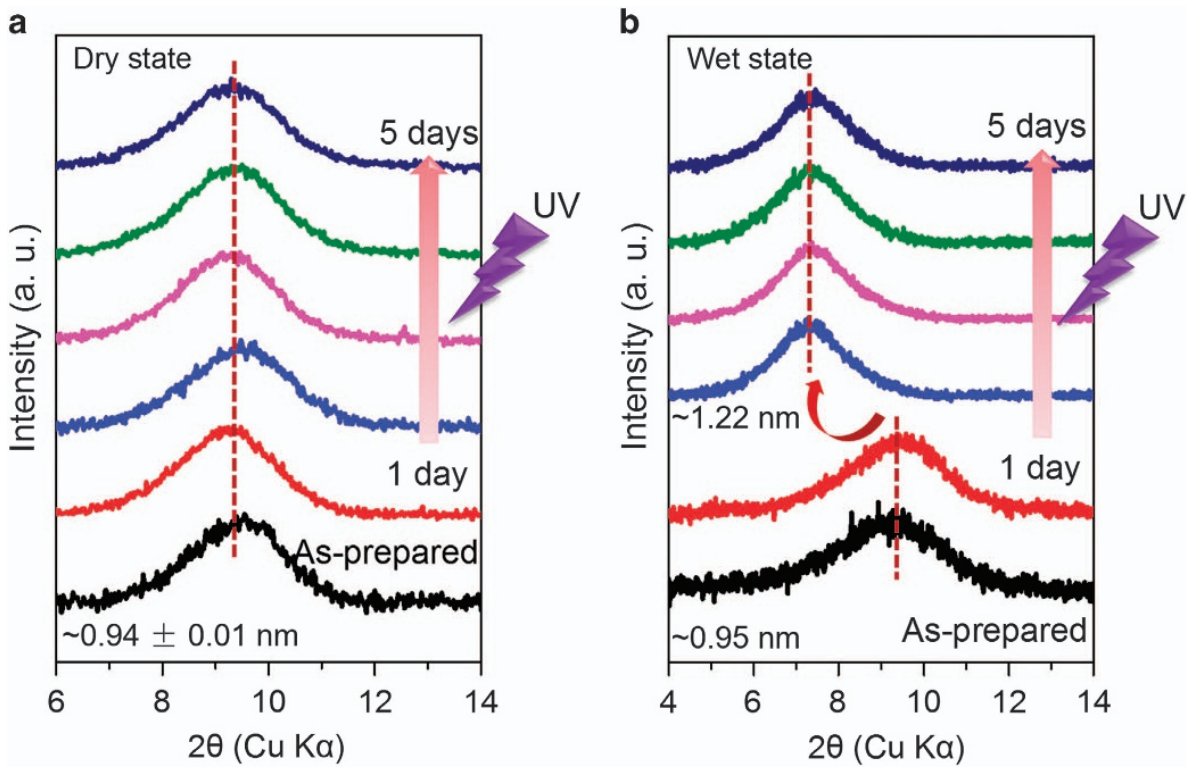

Figure 5 X-ray diffraction analyses of G00.1/TO0.08 membranes after ultraviolet (UV) irradiation for 0-5 days in the (a) dry state and (b) wet state.

rejection for the 3-day UV reduction case can be up to $\sim 95 \%$ compared with the unreduced case. These results indicate that the RGO/TO membranes exhibit excellent salt rejection performances while the high water permeation abilities can be retained, which is favorable for water desalination.

Finally, the mechanism for the excellent desalination and water permeation capabilities of the RGO/TO hybrid membranes is discussed, as illustrated in Figure 1g. By preparing the GO/TO membranes by vacuum-filtrating their hybrid solutions, the GO and TO sheets can be well intercalated into each other to form a uniform lamellar structure, as demonstrated by our previous study ${ }^{34}$ and presented in Figures 1f, $g$ and 5. During the UV irradiation of the $\mathrm{GO} / \mathrm{TO}$ laminates, the GO flakes within the membranes can be reduced mildly to RGO due to the excellent photocatalytic properties of the TO nanosheets (Figures $1 \mathrm{~h}$ and 4). The amount of oxygencontaining functional groups decorated on the GO surfaces is 
a

Water permeation vs. ion permeation

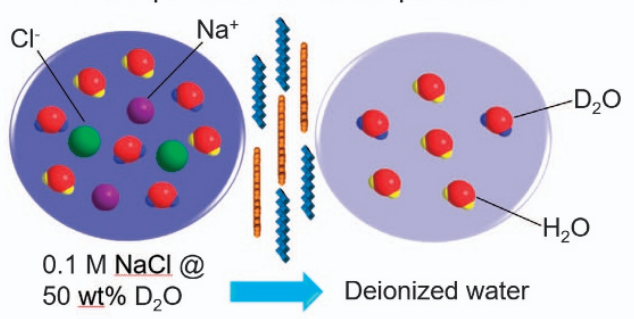

b

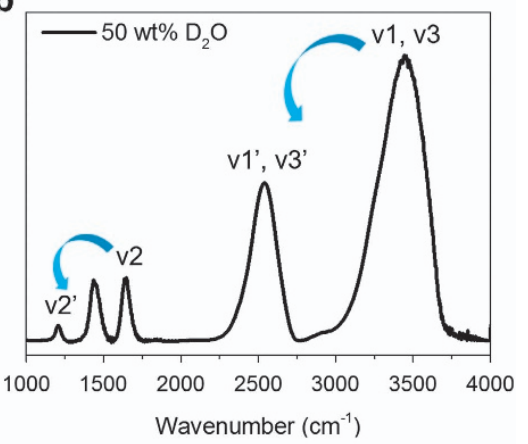

d
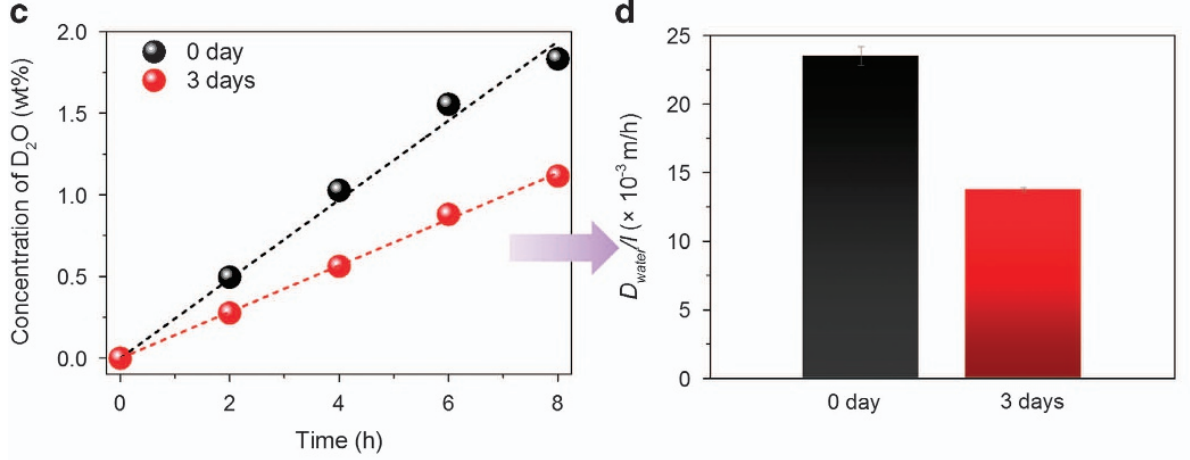

e

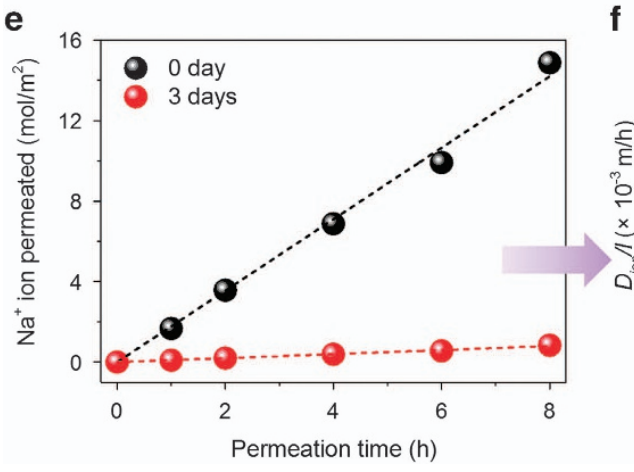

f

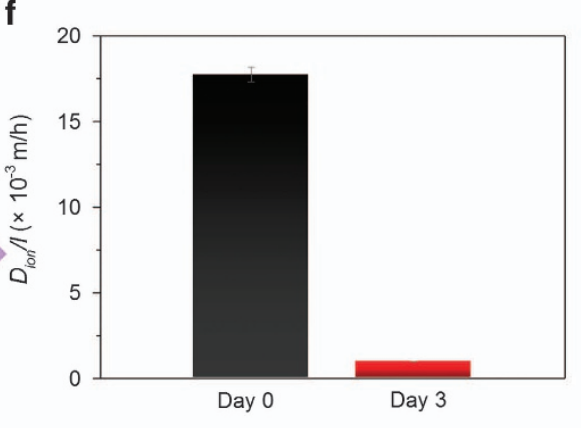

Figure 6 (a) Schematic diagram for the investigation of ion and water permeations through graphene oxide (GO)(RGO)/titania (TO) membranes based on isotope labeling. (b) Fourier transform infrared spectrum of $50 \mathrm{wt} \%$ deuterium oxide $\left(\mathrm{D}_{2} \mathrm{O}\right)$ solution, which indicates the peaks corresponding to $\mathrm{H}_{2} \mathrm{O}$ and $\mathrm{D}_{2} \mathrm{O}$. (c, d) $\mathrm{D}_{2} \mathrm{O}$ permeations and the corresponding water diffusion coefficients through GO/TO membranes after ultraviolet (UV) irradiation for 0 and 3 days, respectively. (e, f) lon permeations and corresponding diffusion coefficients through GO/TO membranes after UV irradiation for 0 and 3 days, respectively.

gradually reduced (Figure 4), which results in a decrease in the local interlayer spacing between the GO flakes and a narrowing of the $s p^{2}$ graphitic nanocapillaries within the laminates, ${ }^{16}$ as illustrated in Figure 1g. These effects may result in an efficient ion rejection of the RGO/TO membranes (Figure 2; Supplementary Figure S2). However, due to the excellent photoinduced hydrophilic conversion of the TO nanosheets under UV irradiation, the wettability of the hybrid membranes is not weakened based on our previous results, ${ }^{32,34}$ and the interlayer spacing can be even enhanced in the wet state (Figure 5). More importantly, the superhydrophilic TO nanosheets intercalated within the membranes can adsorb more water molecules into the membranes and facilitate water transmembrane permeations, which leads to the preservation of the relatively high water permeabilities.

\section{CONCLUSION}

In summary, by intercalating the monolayer TO nanosheets into the GO laminates with mild UV irradiation, excellent water desalination performances can be achieved. For the GO0.1/TO0.08 hybrid membranes, after 3-day UV irradiation, the salt rejection can be up to $95 \%$ compared with the pristine case, whereas the water permeation can be retained to $\sim 60 \%$. The mechanism for the excellent water desalination properties of the RGO/TO membranes is discussed, which indicates that the photoreduction of the GO by TO under UV irradiation is responsible for the efficient salt rejection, whereas the photoinduced hydrophilic transformation of the TO nanosheets within the membranes is responsible for the preservation of the relatively high water permeabilities. These excellent properties provide the RGO/TO hybrid membranes with great potential for practical water desalination. However, there is still a long way to go to achieve their use in practical large-scale applications. For example, although the current RGO/TO hybrid membranes with cellulose microfilters underneath are robust enough for direct manipulation, the mechanical strength of the membranes should be further enhanced to resist high pressures. Fortunately, several promising methods have been recently proposed 
to enhance the mechanical properties of the GO membranes on a large scale, which can also be applied to enhance the mechanical strength of the GO/TO membranes, such as chemical cross-linking by metal ions ${ }^{38}$ and electron-irradiation-induced reinforcement. ${ }^{39}$ Currently, all the experiments are conducted without external high pressures, which are required in practical water desalination applications to enhance the water flux. Moreover, the long time stability of RGO/TO membranes in aqueous environments remains unclear. The efforts on these aspects are in progress and will appear elsewhere in the future. To conclude, the RGO/TO hybrid membranes exhibit excellent salt rejection performances while their relatively high water permeability can be preserved. The results presented in this study may thus lay a foundation for the use of RGO/TO membranes in practical water desalination applications.

\section{ACKNOWLEDGEMENTS}

This work was supported by the Beijing Science and Technology Program (D141100000514001), the National Science Foundation of China (51372133), the National Program on Key Basic Research Project (2014CB932401, 2011CB013000) and the Training Program of Innovation and Entrepreneurship for Undergraduates (201410003B046).

1 Shannon, M. A., Bohn, P. W., Elimelech, M., Georgiadis, J. G., Marinas, B. J. \& Mayes, A. M. Science and technology for water purification in the coming decades. Nature 452, 301-310 (2008)

2 Logan, B. E. \& Elimelech, M. Membrane-based processes for sustainable power generation using water. Nature 488, 313-319 (2012).

3 Novoselov, K. S., Geim, A. K., Morozov, S. V., Jiang, D., Zhang, Y., Dubonos, S. V., Grigorieva, I. V. \& Firsov, A. A. Electric field effect in atomically thin carbon films. Science 306, 666-669 (2004).

4 Bunch, J. S., Verbridge, S. S., Alden, J. S., Zande, A. M., Parpia, J. M., Craighead, H. G. \& McEuen, P. L. Impermeable atomic membranes from graphene sheets. Nano Lett. 8, 2458-2462 (2008)

5 Koenig, S. P., Wang, L., Pellegrino, J. \& Bunch, J. S. Selective molecular sieving through porous graphene. Nat. Nanotechnol. 7, 728-732 (2012).

6 Sint, K., Wang, B. \& Kral, P. Selective ion passage through functionalized graphene nanopores. J. Am. Chem. Soc. 130, 16448-16449 (2008).

7 Cohen-Tanugi, D. \& Grossman, J. C. Water desalination across nanoporous graphene. Nano Lett. 12, 3602-3608 (2012).

8 Hummers, W. S. \& Offeman, R. E. Preparation of graphite oixde. J. Am. Chem. Soc. 80 1339 (1958).

9 Dikin, D. A., Stankovich, S., Zimney, E. J., Piner, R. D., Dommett, G. H., Evmenenko, G., Nguyen, S. T. \& Ruoff, R. S. Preparation and characterization of graphene oxide paper. Nature 448, 457-460 (2007).

10 Eda, G., Fanchini, G. \& Chhowalla, M. Large-area ultrathin films of reduced graphene oxide as a transparent and flexible electronic material. Nat. Nanotechnol. 3 270-274 (2008).

11 Huang, X., Qi, X., Boey, F. \& Zhang, H. Graphene-based composites. Chem. Soc. Rev. 41, 666-686 (2012).

12 Huang, X., Yin, Z., Wu, S., Qi, X., He, Q., Zhang, Q., Yan, Q., Boey, F. \& Zhang, H. Graphene-based materials: synthesis, characterization, properties, and applications. Small 7, 1876-1902 (2011).

13 Tan, C., Huang, X. \& Zhang, H. Synthesis and applications of graphene-based noble metal nanostructures. Mater. Today 16, 29-36 (2013).

14 Loh, K. P., Bao, Q., Eda, G. \& Chhowalla, M. Graphene oxide as a chemically tunable platform for optical applications. Nat. Chem. 2, 1015-1024 (2010).

$15 \mathrm{Eda}, \mathrm{G}$. \& Chhowalla, M. Chemically derived graphene oxide: towards large-area thin-film electronics and optoelectronics. Adv. Mater. 22, 2392-2415 (2010).

16 Nair, N. N., Wu, H. A., Jayaram, P. N., Grigorieva, I. V. \& Geim, A. K. Unimpeded permeation of water through helium-leak-tight graphene-based membranes. Science 335, 442-444 (2012).

17 Boukhvalov, D. W., Katsnelson, M. I. \& Son, Y. W. Origin of anomalous wate permeation through graphene oxide membrane. Nano Lett. 13, 3930-3935 (2013).
18 Joshi, R. K., Carbone, P., Wang, F. C., Kravets, V. G., Su, Y., Grigorieva, I. V., Wu, H. A. Geim, A. K. \& Nair, R. R. Precise and ultrafast molecular sieving through graphene oxide membranes. Science 343, 752-754 (2014).

19 Sun, P., Zhu, M., Wang, K., Zhong, M., Wei, J., Wu, D., Xu, Z. \& Zhu, H. Selective ion penetration of graphene oxide membranes. ACS Nano 7, 428-437 (2013).

20 Sun, P., Zheng, F., Zhu, M., Song, Z., Wang, K., Zhong, M., Wu, D., Little, R. B., Xu, Z. \& Zhu, H. Selective trans-membrane transport of alkali and alkaline earth cations through graphene oxide membranes based on cation- $\pi$ interactions. ACS Nano 8, 850-859 (2014).

21 Sun, P, Wang, K., Wei, J., Zhong, M., Wu, D. \& Zhu, H. Effective recovery of acids from iron-based electrolytes using graphene oxide membrane filters. J. Mater. Chem. A 2, 7734-7737 (2014).

22 Sun, P., Zheng, F., Zhu, M., Wang, K., Zhong, M., Wu, D. \& Zhu, H. Realizing synchronous energy harvesting and ion separation with graphene oxide membranes. Sci. Rep. 4, 5528 (2014).

$23 \mathrm{Hu}, \mathrm{M}$. \& Mi, B. Enabling graphene oxide nanosheets as water separation membranes. Environ. Sci. Technol. 47, 3715-3723 (2013).

$24 \mathrm{Han}$, Y., Xu, Z. \& Gao, C. Ultrathin graphene nanofiltration membrane for water purification. Adv. Funct. Mater. 23, 3693-3700 (2013).

$25 \mathrm{Mi}$, B. Graphene oxide membranes for ionic and molecular sieving. Science 343 740-742 (2014).

26 Su, Y., Kravets, V. G., Wong, S. L., Waters, J., Geim, A. K. \& Nair, R. R. Impermeable barrier films and protective coatings based on reduced graphene oxide. Nat. Commun. 5, 4843 (2014).

$27 \mathrm{Ma}, \mathrm{R}$. \& Sasaki, T. Nanosheets of oxides and hydroxides: ultimate 2D charge-bearing functional crystallites. Adv. Mater. 22, 5082-5104 (2010).

28 Wang, L. \& Sasaki, T. Titanium oxide nanosheets: graphene analogues with versatile functionalities. Chem. Rev. 114, 9455-9486 (2014).

29 Sasaki, T., Watanabe, M., Hashizume, H., Yamada, H. \& Nakazawa, H. Macromoleculelike aspects for a colloidal suspension of an exfoliated titanate. Pairwise association of nanosheets and dynamic reassembling process initiated from it. J. Am. Chem. Soc. 118, 8329-8335 (1996).

30 Sasaki, T. \& Watanabe, M. Osmotic swelling to exfoliation. Exceptionally high degrees of hydration of a layered titanate. J. Am. Chem. Soc. 120, 4682-4689 (1998).

31 Williams, G., Seger, B. \& Kamat, P. V. TiO ${ }_{2}$-graphene nanocomposites. UV-assisted photocatalytic reduction of graphene oxide. ACS Nano 2, 1487-1491 (2008).

32 Sun, P., Zhu, M., Ma, R., Wang, K., Wei, J., Wu, D., Sasaki, T. \& Zhu, H. Graphene oxide/titania hybrid films with dual-UV-responsive surfaces of tunable wettability. RSC Adv. 2, 10829-10835 (2012)

33 Sakai, N., Fukuda, K., Shibata, T., Ebina, Y., Takada, K. \& Sasaki, T. Photoinduced hydrophilic conversion properties of titania nanosheets. J. Phys. Chem. B 110, 6198-6203 (2006).

34 Sun, P., Ma, R., Wang, K., Zhong, M., Wei, J., Wu, D., Sasaki, T. \& Zhu, H. Suppression of the coffee-ring effect by self-assembing graphene oxide and monolayer titania. Nanotechnology 24, 075601 (2013).

35 Akhavan, O., Abdolahad, M., Esfandiar, A. \& Mohatashamifar, M. Photodegradation of graphene oxide sheets by $\mathrm{TiO}_{2}$ nanoparticles after a photocatalytic reduction. J. Phys. Chem. C 114, 12955-12959 (2010).

36 Wang, Y., Sun, C., Yan, X., Xiu, F., Wang, L., Smith, S. C., Wang, K. L., Lu, G. Q. \& Zou, J. Lattice distortion oriented angular self-assembly of monolayer titania sheets. J. Am. Chem. Soc. 133, 695-697 (2011).

37 Sun, P., Liu, H., Wang, K., Zhong, M., Wu, D. \& Zhu, H. Ultrafast liquid water transport through graphene-based nanochannels measured by isotope labelling. Chem. Commun. 51, 3251-3254 (2015)

38 Park, S., Lee, K.-S., Bozoklu, G., Cai, W., Nguyen, S. T. \& Ruoff, R. S. Graphene oxide papers modified by divalent ions - enhancing mechanical properties via chemical crosslinking. ACS Nano 2, 572-578 (2008).

39 Jin, E., He, J., Sheng, K., Zhang, Z., Shi, G. \& Zheng, Q. Electron-irradiation-induced reinforcement of reduced graphene oxide papers. Acta Mater. 61, 6466-6473 (2013)

(c) (1)(5) This work is licensed under a Creative Commons Attribution-NonCommercial-ShareAlike $\quad 4.0$ International License. The images or other third party material in this article are included in the article's Creative Commons license, unless indicated otherwise in the credit line; if the material is not included under the Creative Commons license, users will need to obtain permission from the license holder to reproduce the material. To view a copy of this license, visit http://creativecommons.org/ licenses/by-nc-sa/4.0/

Supplementary Information accompanies the paper on the NPG Asia Materials website (http://www.nature.com/am) 